\title{
Effect of Ballistic Bouncing of Gas Particles across a Microchannel on Rarefied Gas Flows
}

\author{
Nikolai Kislov \\ Nano CVD Company, Tampa, Florida, USA \\ Email: nikolai95@verizon.net
}

How to cite this paper: Kislov, N. (2021) Effect of Ballistic Bouncing of Gas Particles across a Microchannel on Rarefied Gas Flows. Journal of Applied Mathematics and Physics, 9, 779-808. https://doi.org/10.4236/jamp.2021.94054

Received: March 24, 2021

Accepted: April 26, 2021

Published: April 29, 2021

Copyright (c) 2021 by author(s) and Scientific Research Publishing Inc. This work is licensed under the Creative Commons Attribution-NonCommercial International License (CC BY-NC 4.0). http://creativecommons.org/licenses/by-nc/4.0/ (c) (7) \& Open Access

\begin{abstract}
This paper proposes a novel computationally efficient method of modeling rarefied gas flow in microchannels based on the newly discovered and mathematically proven Ballistic Principle of the Property Balance in Space (BPPBS). The mechanism of influence of the effect of rarefication on the gas flow is specifically investigated. Also, a differential form of the momentum balance equation governing gas flow in the channel between two parallel plates due to the pressure gradient along the channel and its exact implicit solution in the form of an integral equation have been derived. The theory does not use the generalized concept of viscosity based on the variable mean free path (MFP) in the Knudsen layer (KL). Comparing the normalized flow rate as a function of the inverse Knudsen number according to the current theory and the experimental data shows good agreement in the range of the inverse Knudsen number from 0.01 to about 40 . The correlation factor is found to be about 0.995 . The results show that our approach based on the BPPBS offers substantial and practical advantages in modeling and simulation of rarefied gases. The validity of the widely disseminated claim of the geometry-dependent MFP in the KL was analyzed.
\end{abstract}

\section{Keywords}

Navier-Stokes, CFD, Gas Flow, Rarefied, Microchannel

\section{Introduction}

The modeling gas flow in microfluidic devices for microelectromechanical systems (MEMS) is critically important. MEMS devices based on parallel-plate structures have often been used as the capacitive sensing, electrostatic actuation mechanisms in micro gyroscopes, accelerometers, switches, mirrors, pressure sensors [1]. In these devices, the gas rarefaction effect can be substantial. There- 
fore, each molecule's interaction with the confining plates must be considered to predict the fluid impact on the device operation accurately.

A well-known method of obtaining analytical solutions for isothermal gaseous flow with slip boundary conditions is based on the locally fully developed flow assumption. The following Navier-Stokes equation governing gas flow in a two-dimensional channel (with $x$-axis along the channel and $y$-axis across the channel between two spaced at distance $H$ parallel plates) is expressed as:

$$
\frac{\partial^{2} u}{\partial y^{2}}=\frac{1}{\eta} \frac{\mathrm{d} p}{\mathrm{~d} x}
$$

and applying the second-order velocity slip boundary conditions (Maxwell-type assumption) in the following form [2]

$$
u-u_{w}= \pm C_{1} \lambda_{f} \frac{\partial u}{\partial y}-C_{2} \lambda_{f}^{2} \frac{\partial^{2} u}{\partial y^{2}},
$$

where $\eta$ is the fluid dynamic viscosity, $u$ is the gas slip velocity near the wall, $u_{w}$ is the tangential velocity of the wall, and $\lambda_{f}$ is the mean free path, $C_{1}$ and $C_{2}$ are constants. It is well-known from the gas kinetic theory the following relationship:

$$
\eta=\frac{1}{2} m n v_{T} \lambda_{f}
$$

where $n$ is gas-particle density, $m$ is gas-particle mass, $V_{T}$ is the magnitude of the thermal velocity, and $\eta$ is the fluid dynamic viscosity in one-dimensional gas space. However, according to [3], the relationship above is "valid for flows in the region far away from the wall". Also, "within the KL, the flight paths of gas molecules are so significantly influenced by the wall that this relation becomes invalid [3]". Knudsen layer (KL), known from the literature as adjacent to a gas-solid interface region of several mean free paths (MFP) thick, and a variable MFP linked to defining a geometry-dependent "viscosity" in the adjacent to the gas-solid interface region are widely used abstractions that helped to explain gas microflow behavior. We continue the introduction by briefly describing some of the theoretical and computational research dealing with the variable MFP and the geometry-dependent "viscosity" and our recent results presented in [4], which were obtained without using the concept of the Knudsen layer at all.

A rarefied gas flow is characterized by using the dimensionless Knudsen number $(K n)$, which is defined as the MFP ratio to a characteristic length scale of the gas flow. Keeping in mind that gas transport takes place through intermolecular collisions separated by the ballistic motion of molecules characterized by the molecular free paths, one can conclude that the MFP is an important parameter in gas dynamics. The KL, adjacent to a gas-solid interface region of several mean free paths thick, is another important rarefaction parameter. It is defined as the region of local nonequilibrium extending a thickness of a few MFPs from the wall in gas microflows [5]. (Note: in the text, the words "wall" and "plate" are identical in defining a gas-solid interface). It is also believed that, in the KL, 
gas molecule-surface collisions are more frequent than gas molecule-molecule collisions, i.e., the gas MFP will effectively be reduced in the KL [6]. It is also suggested that " $[\mathrm{i}] \mathrm{n}$ a rarefied gas system, in which gas molecules may not suffer sufficiently frequent collisions with other gas molecules to attain equilibrium conditions, deviations from thermodynamic equilibrium may have substantial effects". The authors of [6] further proposed a power-law (PL) form for the probability distribution function for nonequilibrium MFP, which is rather phenomenological than a physically justified hypothesis.

The variety of recent studies considers the collisions between the freely moving gas molecules and wall atoms since they believed that these collision events lead to the momentum change, and show employing molecular dynamics simulation (MD) that the MFP varies near surfaces [7] [8] [9] [10]. However, one primary limitation of the MD simulation is that it is only valid for the free molecular regime $(K n>10)$. The MD simulation does not account for interactions between molecules. Another limitation of the code is that model systems for engineering applications require hundreds of thousands of particles; it is considered impossible to analytically determine such systems' properties. Therefore, the problem must be solved by using numerical methods [11]. Unfortunately, the MD simulation is computationally very intensive. Also, long MD simulations are mathematically ill-conditioned, generating cumulative errors in numerical integration [11]. Then, there has been significant effort through the years to expand the continuum fluid dynamics description to the transition regime $(0.1 \leq$ $K n \leq 10)$. One common approach consists of using an effective viscosity near the wall, which is integrated into the existing models of a viscous gas flow and operated in a CFD arrangement based on the classical Navier-Stokes equations. As in the elementary kinetic theory, the viscosity is directly proportional to the molecular mean free path (MFP). The effective gas viscosity is defined similarly in the near-wall region [12] and [13].

In this approach, a wall is included in the system so that some molecules will hit the wall, and the wall will terminate their flight paths. Consequently, the MFP of all the molecules in the system may be smaller than the MFP in the unconfined gas space because of the boundary limiting effect [12] [13]. More specifically, in the most recent study, Abramov [12] introduced a new expression for the MFP of gases in the KL. First, he used a known from the kinetic theory for an ideal gas a probability distribution function $f(r)$ of the length $r$ of the free flight until a collision in the form

$$
f(r)=\frac{1}{\lambda_{0}} \mathrm{e}^{-r / \lambda_{0}},
$$

where $\lambda_{0}$ is the MFP in an unbounded space.

Second, he defined the expectation $E\left(r_{0}\right)$ "of the length $r$ of the free flight until a collision either with another molecule, or, if none happens, with the obstacle upon covering the distance $r_{0}$ ". It is resulted in finding that

$$
\lambda\left(r_{0}\right)=E\left(r_{0}\right)=\lambda_{0}\left(1-\mathrm{e}^{-r_{0} / \lambda_{0}}\right)
$$


Finally, Abramov [12] integrated Equation (5) over the azimuthal and polar angles of the direction of the flight of a suitable spherical coordinate system to obtain the dependence of the MFP as a function of the distance $d$ from a wall, which has resulted in the following:

$$
\lambda(d)=\lambda_{0}\left(1-\frac{1}{2}\left(\mathrm{e}^{-d / \lambda_{0}}-\frac{d}{\lambda_{0}} E_{1}\left(\frac{d}{\lambda_{0}}\right)\right),\right.
$$

where $E_{1}$ is a standard notation for the exponential integral

$$
E_{1}\left(\frac{d}{\lambda_{0}}\right)=\int_{\frac{d}{\lambda_{0}}}^{\infty} \frac{\mathrm{e}^{-y}}{y} \mathrm{~d} y .
$$

Hadjiconstantinou [14] noted that "for $K n \leq 0.1$, kinetic effects are limited to the vicinity of the walls, one can obtain solutions of the linearized Boltzmann equation by superposing a Navier-Stokes solution with a kinetic boundary (Knudsen) layer correction, where the latter is significant only up to distances of the order of one mean free path from the wall". However, Guo et al. [3] extended the applicability of the Navier-Stokes equations to much higher Knudsen numbers (up to about 80, see Figure 4 in [3]). They claimed that because the data of their extended Navier-Stokes constitution (ENSC) model "agree well with the experimental data in the entire range of $\delta$, and the Knudsen minimum in the flux is captured successfully", their model of the effective viscosity is valid. Still, it is difficult to imagine the applicability of the Navier-Stokes equations, which are designed to describe fluid flow in the infinite small volume, to the rarefied gas media having a gas-particle density low as one gas particle per 10 - 80 distances between the walls. It is also disputed if such behavior of the effective MFP near the wall is physical or not [15]".

The authors of this study admitted "erroneous definition and calculation practices." They also highlighted that "variations in MFP as a function of the surface confinement, which disagrees with the kinetic theory and leads to wrong physical interpretations of nanoscale gas flows. This controversy occurs due to erroneous definition and calculation practices, such as consideration of gas wall collisions, using local bins smaller than a MFP, and utilizing time frames shorter than a mean collision time in the MFP calculations". They also stated that the collisions between the moving gas molecules and the solid wall should be ignored when evaluating the individual free paths. Finally, they highlighted that "[1]ocal MFP variations are physically admissible only if there are local density and temperature variations in the system".

Another approach consists in revisiting "the problem of micro-channel compressible gas flows and show[ing] that the axial diffusion of mass engendered by the density (pressure) gradient becomes increasingly significant with increased Knudsen number compared to the pressure-driven convection [16]." Dongari et al. [16] used "a recently proposed modification of the Navier-Stokes equations that include the diffusion of mass caused by the density and temperature gradients [17]". Dongari et al's approach has successfully captured the Knudsen 
paradox, which "relates to the presence of a minimum when the mass flow rate normalized by the pressure difference is plotted against the Knudsen number [16]." Also, they demonstrated "that the normalized volume flow rate reaches a constant value for $K n \rightarrow \infty$ which is in turn in agreement with the Knudsen's finding". However, their findings are applicable only for compressible gases. The total mass flow rate for compressible gases through a micro-channel is expressed as the sum of the convective and self-diffusive mass transport. In Dongari et al.'s interpretation, Knudsen's minimum is "clarified based on the inclusion of axial diffusion. The occurrence of minimum flux is caused by the fact that the diffusive mode of transport begins to dominate the convective mode of transport at the higher Knudsen numbers". It implies that if one accepts the explanation above, the Knudsen paradox would not exist for incompressible gases.

Returning to the critical notes in [15], we also believe that the MFP is a function of the free flight distance that leads to a wrong physical interpretation of the MFP. In a "small-scale" view, a particle directed toward a wall is not aware of its possible collision with the wall during its free flight. Therefore, it may interact by collision with nearby particles with the same frequency as if it travels in the unbounded gas space unless a near-wall gas density is higher than the bulk gas density. Moreover, while recent literature presents a lot of theoretical and computational research dealing with the momentum transfer across the channel (the momentum associated with the gas flow along the channel), it is silent about the balance across the channel of both the mass and momentum associated with the thermal velocity component. Violation of these balances will lead to disagreement with the kinetic theory of gases and eventually produce an erroneous physical interpretation of microscale gas flows and, therefore, need to be verified.

Our recent publications [4] and [17] support some of the arguments in [15]. In these studies, we discuss a novel analytical molecular dynamics technique based on a newly discovered Ballistic Principle of the Property Balance in the Space (BPPBS) occupied by the gas. According to the BPPBS, in each nonmoving point of the gas space at a given time, the net rate of property (mass, momentum, or energy) influx per unit volume, formed by the converging ballistic particles (each traveling along a ballistic trajectory with certain survival probability) from the model gas system is equated to the temporal rate of property change per unit volume and the net rate of property efflux per unit volume, formed by the diverging ballistic particles. Moreover, each of the balances (mass, momentum, or energy) shall be maintained independently for any point of the gas space at any given time. In paper [4], we have validated the BPPBS by seven validation tests. The most important of them was demonstrating that, in the collision-dominated flow regime, the differential equations, which we converted from the derived integro-differential mass and momentum balance equations, are identical to the corresponding Navier-Stokes equations. This finding supports the assumption that the formulated integro-differential forms of the balance are exact implicit solutions for corresponding Navier-Stokes equations. 
One more of the validation tests included analysis and verification of the mass balance and momentum balance in a steady-state incompressible gas system confined by gas-solid interfaces with a purely diffuse scattering of particles at the uniform temperature. In such a system, all variables describing flow depend on the position in gas space and are not dependent on time so that the particle density $n$ and associated with it the MFP, the mass $m$ and the magnitude of the thermal velocity of the particle/molecule, $V_{T}$, are constant. We have verified that, in each point of space between confining parallel plates, the mass and momentum balance are conserved. It implies that any deviation from the constant particle density or the MFP will violate the BPPBS and violate the physical principles.

Additionally, as we referenced above, the flow of a dilute gas near a solid surface is usually explained in various theories by non-continuum effects assumed to exist in the Knudsen layer. In contrast, our approach does not use the concept of the Knudsen layer at all. Particularly, using basic principles of Newton's mechanics and the kinetic theory of gases, we have derived an analytical representation of the gas flow velocity profile in the microchannel formed by two being at rest infinite parallel plates spaced at distance $H$ (see Equation (116) of [4]) given below:

$$
\boldsymbol{u}_{x}(y)=-\frac{H}{m n v_{T}} \frac{\mathrm{d} P}{\mathrm{~d} x} \frac{1}{K n}\left(-\frac{y^{2}}{H^{2}}+\frac{y}{H}+\frac{2-\sigma}{\sigma} K n+2 K n^{2}\right),
$$

where $n$ is the particle density, $m$ is the mass, $V_{T}$ is the magnitude of the thermal velocity of the particle/molecule, $\sigma$ is the momentum accommodation coefficient, which is the probability, for an incident particle, to accommodate momentum from the gas-solid interface and to scatter back in the model gas as a diffuse particle, $K n=\frac{\lambda_{f}}{H}$ is the Knudsen number, $\lambda_{f}$ is the mean free path, and $\frac{\mathrm{d} P}{\mathrm{~d} x}$ is the pressure gradient forcing gas flow along the channel with the velocity distribution $\boldsymbol{u}_{x}(y)$ across the channel. Remarkably, the derived tangential slip velocity coefficient (the third right-hand term in parenthesis) does contain the term being proportional to $\frac{2-\sigma}{\sigma}$. Its appearance is not the result of using the semi-empirical Maxwell-type assumptions or the Knudsen layer's notion but the result of the application of the BPPBS.

Also, we compared the non-dimensional flow rates calculated by Equation (110) of [4] and rescaled by $\sqrt{\pi} / 2$ factor and a selection of slip models proposed by various authors [19] [20] [21] [22] [23]. Equation (110) of [4] reveals there is a minimum in the normalized mass flow rate (at about $K n \cong 0.3$ ), which is called the Knudsen paradox or Knudsen minimum in the literature. There is no discrepancy between our Ballistic Model and various slip models at $K n<0.1$. However, while the results obtained according to the Ballistic Model of [4] are close to the results of Shen et al. [20] by BGK model or Li et al. [22], they differentiate significantly at $K n>0.3$ for the other models. Still, the re- 
sults obtained according to our Ballistic Model are within the reasonable range of discrepancies of a variety of the literature data (see also Table 6 and Figure 7 in [24]). Again, the Knudsen minimum's appearance in the normalized volume flow rate results from neither usage of a geometry-dependent "viscosity" being generalized from variable local MFP near the gas-solid interface, nor the semi-empirical Maxwell-type assumptions but the result of the application of the BPPBS.

This paper further promotes the newly discovered BPPBS and its application to describe steady-state fluid flows in a microchannel. It specifically investigates the mechanism of influence of the Knudsen number on the rarefied gas flow. It offers a new way of computing a steady-state rarefied gas flow in a microchannel, which does not use the generalized concept of viscosity based on the variable MFP in the KL.

In Section 2 of this paper, we further analyze the validity of the currently widely disseminated claim of the MFP variation normal to the nano-channel surfaces as a function of the confinement level. We applied validation test 4.1, "Determining the total rate of collisions per unit area on a surface being in contact with the gas" of [4], for evaluation of how the MFP variation normal to the nano-channel surface claimed in [12] affects the rate of collisions per unit area. It also provides a quantitative measure of how these results differentiate from corresponding the collisions' rate per unit area of an ideal gas according to the kinetic theory of gases.

Section 3 describes the properties and features of the model gas, the Ballistic Model's physical principles, and the general physical principles of constructing the property balance for one-dimensional steady-state gas flow.

In Section 4, we provide integro-differential forms of mass balance and momentum balance equations formulated for the gas space bounded by two infinite parallel plates.

In Section 5, we demonstrate the effect of ballistic bouncing of gas particles between confining plates on rarefied gas flows.

Section 6 shows a way to obtaining a differential form $u_{x}$-momentum balance equation governing gas flow in the channel between two parallel plates due to the pressure gradient along the channel.

Finally, in Section 7, we present the discussion and conclusions and highlight the advantages of the proposed approach for modeling rarefied-gas flows or gas flows in MEMS.

\section{Evaluation of the Claim about Spatial Variation of MFP near a Wall}

In our recent publications [4] and [18], we used several validation tests to evaluate the feasibility of the BM and the BPPBS. Particularly, we determined two gas properties: the rate of collisions per unit area and the pressure exerted on a solid wall contacting with the incompressible gas. We have found that both our 
results and the corresponding results from the kinetic theory of gases are correlated and analytically identical. These findings support the BM and the BPPBS. Here we use the same approach in evaluating the claim about spatial variation of MFP near a wall claimed in [12].

\subsection{Determining the Total Rate of Collisions Per Unit Area on a Surface Being in Contact with the Gas in a Three-Dimensional Configuration}

Figure 1 is a perspective view of the geometry and coordinate system for determining the total rate of collisions per unit area on a planar flat surface being in contact with the gas. In a system with no gravitational force, the ballistic particles have straight-line trajectories. Here our consideration is limited to an incompressible model gas at the uniform temperature in a steady-state condition. However, following [12], we assume the spatial variation of MFP near a wall expressed analytically by Equation (5). We plan to verify whether the notion of the variable MFP near a wall is physically justified.

In a semi-sphere filled with the incompressible gas over the being at rest surface $A_{s}$ having a directional vector $\overrightarrow{\boldsymbol{n}}$, each particle having an instantaneous randomly directed vector of the thermal velocity of magnitude, $V_{T}$ may have the instant vector-velocity component directing a particle toward the surface $A_{s}$ In Figure 1 the particle 101 positioned at $\overrightarrow{\boldsymbol{r}}^{\prime}$ is shown at distance $y^{\prime}$ from the surface $A_{s^{\prime}}$ The angle between the instant vector $\overrightarrow{\boldsymbol{n}}_{i 0}$ and the directional vector $\overrightarrow{\boldsymbol{n}}$ is labeled as $\theta$. The ends of the directionally random vector-velocity of magnitude $v_{T}$ form spherical surface 102 .

Adopting Equation (34) of [4] to the conditions above and assigning $\Psi_{i n}=1$, the total rate of collisions per unit area on the surface $A_{s}$, is given as:

$$
Z=-\frac{1}{4 \pi} \iiint_{V} \frac{Z_{V}\left(\overrightarrow{\boldsymbol{r}}^{\prime}\right)}{\left|\overrightarrow{\boldsymbol{r}}-\overrightarrow{\boldsymbol{r}}^{\prime}\right|^{2}} Q_{i 0}\left(\overrightarrow{\boldsymbol{r}}, \overrightarrow{\boldsymbol{r}}^{\prime}\right) \overrightarrow{\boldsymbol{n}}_{i 0} \cdot \overrightarrow{\boldsymbol{n}} \mathrm{d} V^{\prime},
$$

where

$$
\overrightarrow{\boldsymbol{n}}_{i 0}=\frac{\overrightarrow{\boldsymbol{r}}-\overrightarrow{\boldsymbol{r}}^{\prime}}{\left|\overrightarrow{\boldsymbol{r}}-\overrightarrow{\boldsymbol{r}}^{\prime}\right|}
$$

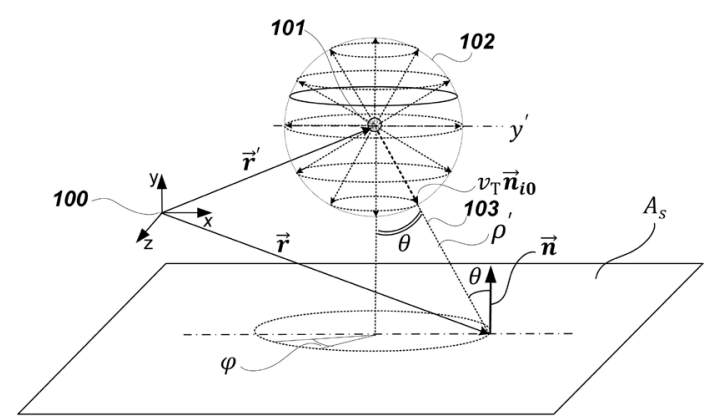

Figure 1. The perspective view of the geometry and coordinate system for determining the total rate of collisions per unit area and the pressure exerted on the surface from the gas volume. 
and $Z_{V}$ is the rate of collisions per unit volume. In [4], we derived $Z_{V}$ by substitution of Equation (25) in Equation (77) of [4], so that $Z_{V}$ is expressed as

$$
Z_{V}=\frac{2}{3} n P_{c} v_{T}
$$

where $n$ is particle density, $V_{T}$ is the magnitude of the thermal velocity, and $P_{c}$ is the number of particles placed within a collision tube of a unit length in an unbounded gas space.

Recognizing the similarity of the geometry of computation in [4] and [12], we point out the identity of the following parameters

$$
d \equiv y ; r_{0} \equiv \rho^{\prime}=\frac{y^{\prime}}{\cos (\theta)} ; \lambda_{0} \equiv \frac{1}{P_{c}} .
$$

In Equation (12), the left-hand terms are from [12], and the right-hand terms are from [4] and Figure 1. Since the number of particles placed within a collision tube of a unit length depends on the particle's trajectory [12], which, as we mentioned above, seems to be a wrong physical interpretation, we define $P_{c}(y, \theta)$ as follows

$$
P_{c}\left(y^{\prime}, \theta\right)=\frac{1}{E\left(r_{0}\right)}=\frac{1}{\lambda_{0}\left(1-\mathrm{e}^{-r_{0} / \lambda_{0}}\right)} \equiv \frac{P_{c}}{\left(1-\mathrm{e}^{-\frac{P_{c} y^{\prime}}{\cos (\theta)}}\right)} .
$$

The number of particles placed within a collision tube of a unit length along trajectory 103 is a function of both the distance from the wall $y$ and the angle of the incidence $\theta$. In Equations (9) and (11), we will use $P_{c}\left(y^{\prime}, \theta\right)$ defined in the equation above as a replacement for $P_{c}$ Still, the probability of free path traveling along the ballistic trajectory 101 is expressed as

$$
Q_{i 0}\left(\overrightarrow{\boldsymbol{r}}, \overrightarrow{\boldsymbol{r}}^{\prime}\right)=\exp \left(-\frac{4}{3} P_{c}(y, \theta) \frac{y^{\prime}}{\cos (\theta)}\right)=\exp \left(-\frac{4}{3} \frac{P_{c}}{\left(1-\mathrm{e}^{-P_{c} y / \cos (\theta)}\right)} \frac{y^{\prime}}{\cos (\theta)}\right)
$$

Finally, using the geometry illustrated in Figure 1, considering that

$$
\left|\overrightarrow{\boldsymbol{r}}-\overrightarrow{\boldsymbol{r}}^{\prime}\right|=\rho^{\prime}=y^{\prime} / \cos (\theta)
$$

and

$$
\overrightarrow{\boldsymbol{n}}_{i 0} \cdot \overrightarrow{\boldsymbol{n}}=-\cos (\theta),
$$

and substituting Equations (11), (13), (15) and (16) in Equation (9), in which $\mathrm{d} V^{\prime}=\rho^{\prime 2} \sin (\theta) \mathrm{d} \theta \mathrm{d} \varphi \mathrm{d} \rho^{\prime}$, we obtain:

$$
\begin{aligned}
Z & \equiv Z_{\beta} \\
& =\frac{2}{3} n P_{c} v_{T} \frac{1}{4 \pi} \int_{0}^{\frac{\pi}{2}} \int_{0}^{2 \pi} \int_{0}^{\infty} \frac{\exp \left(-\frac{4}{3} \frac{P_{c}}{\left(1-\beta \mathrm{e}^{-P_{c} y^{\prime} \cos (\theta)}\right)} \frac{y^{\prime}}{\cos (\theta)}\right)}{\left(1-\beta \mathrm{e}^{-P_{c} y^{\prime} / \cos (\theta)}\right)} \sin (\theta) \mathrm{d} \theta \mathrm{d} \varphi \mathrm{d} y^{\prime}
\end{aligned}
$$

where $\beta$ is the model parameter. For the BM (compare with Equation (81) of 
[4]), $\beta=0$. One can easily perform integration of Equation (17), in which $\beta=0$, which is calculated as

$$
Z_{\beta=0}=\frac{2}{3} n P_{c} v_{T} \frac{1}{4 \pi} \int_{0}^{\frac{\pi}{2}} \int_{0}^{2 \pi} \int_{0}^{\infty} \exp \left(-\frac{4}{3} P_{c} \frac{y^{\prime}}{\cos (\theta)}\right) \sin (\theta) \mathrm{d} \theta \mathrm{d} \varphi \mathrm{d} y^{\prime}=n \frac{v_{T}}{4}
$$

The result of integration in Equation (18) is identical to the rate of collisions per unit area of an ideal gas according to the kinetic theory of gases, thus supporting the Ballistic Model's validity. However, modification of the scheme of calculation by incorporation of the corresponding expression for the MFP derived in [12] has led to the dependence (see Equation (17) in which $\beta=1$ ), which is not in agreement with the kinetic theory of gases.

\subsection{Determining the Total Rate of Collisions Per Unit Area on a Surface Being in Contact with the Gas in a One-Dimensional Configuration}

For clarity and simplicity of quantitative evaluation, we provide below the analysis of a one-dimensional gas system. Figure 2 is a planar view of the geometry of a one-dimensional gas system for determining the total rate of collisions per unit area on the planar plate 201 from the gas volume. The ballistic particles have straight-line trajectories toward and outward the plate in the system with no gravitational force. Here again, we consider an incompressible gas at the uniform temperature in a steady-state condition. In a space filled with the incompressible gas over the being at rest plate 201 having a directional vector $\boldsymbol{n}=+1$, each particle 202 having an instantaneous randomly directed vector of the thermal velocity of magnitude $v_{T}$ may have the instant vector-velocity component $\boldsymbol{n}_{i 0}=-1$ directing a particle toward the plate 201. In Figure 2, the particle 202 positioned at $y^{\prime}$ is shown at distance $y^{\prime}$ from plate 201 .

Upon modification of Equation (9) to applying to the one-dimensional configuration, the total rate of collisions per unit area on plate 201 (schematically shown by arrow 203) is given as:

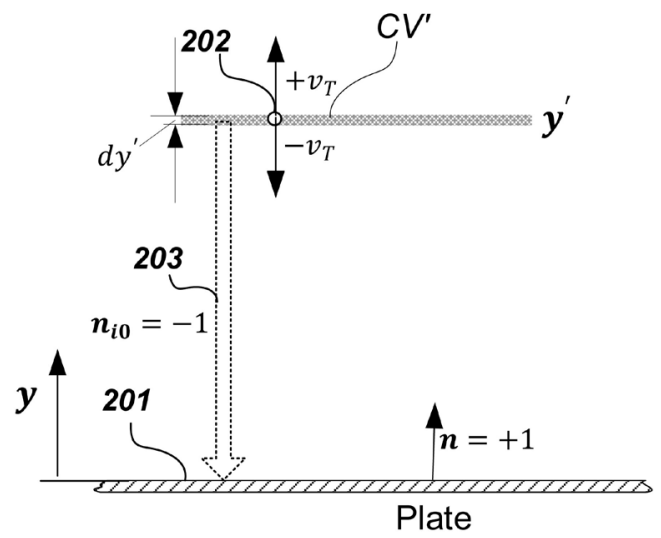

Figure 2. The planar view of the geometry of a one-dimensional gas system for determining the total rate of collisions per unit area on the planar flat surface from the gas volume. 


$$
Z_{b}=-\frac{1}{2} \int_{0}^{\infty} Z_{V}\left(y^{\prime}\right) Q_{i 0}\left(y^{\prime}, 0\right) \boldsymbol{n}_{i 0} \boldsymbol{n} \mathrm{d} y^{\prime},
$$

where $Z_{V}$ is the rate of collisions per unit volume and $Q_{i 0}$ is the probability of free path traveling from the control volume $C V^{\prime}$ at $y^{\prime}$ to the plate 201. In [4], we defined $Z_{V}$ for one-dimensional incompressible gas as follows

$$
Z_{V}=\frac{1}{2} n P_{c} v_{T}
$$

where $n$ is gas-particle density, $V_{T}$ is the magnitude of the thermal velocity, and $P_{c}$ is the number of particles placed within a collision tube of a unit length in an unbounded gas space. The survival probability $Q_{i 0}\left(y^{\prime}, 0\right)$ that a particle will have traveled along the ballistic trajectory in incompressible model gas at the uniform temperature from $y^{\prime}$ to $y=0$ is calculated as

$$
Q_{i 0}\left(y^{\prime}, 0\right)=\exp \left(-P_{c} y^{\prime}\right)
$$

Substituting Equations (20) and (21) in Equation (19) and executing integration, we obtain

$$
Z=\frac{1}{4} n P_{c} v_{T} \int_{0}^{\infty} \exp \left(-P_{c} y^{\prime}\right) \mathrm{d} y^{\prime}=\frac{1}{4} n v_{T}
$$

The result of integration in Equation (22) is identical to the derivation of the rate of collisions per unit area of an ideal gas according to the kinetic theory of gases, thus supporting the Ballistic Model's validity.

We use the same approach to evaluate the rate of collisions per unit area of the gas, in which spatial variation of MFP near a wall [12] exists. Modifying Equation (13) so that it will be applicable for one-dimensional gas configuration, we obtain

$$
P_{c}\left(y^{\prime}, \theta=0\right)=P_{c}\left(y^{\prime}\right)=\frac{P_{c}}{1-\mathrm{e}^{-P_{c} y^{\prime}}} .
$$

In Equations (20) and (21), we will use $P_{c}\left(y^{\prime}\right)$ defined in the equation above as a replacement for $P_{c}$. Substituting Equations (20) and (21) modified by Equation (23) in Equation (19), we obtain

$$
Z=\frac{1}{4} n v_{T} \int_{0}^{\infty} \frac{P_{c}}{1-\mathrm{e}^{-P_{c} y^{\prime}}} \exp \left(-\frac{P_{c}}{1-\mathrm{e}^{-P_{c} y^{\prime}}} y^{\prime}\right) \mathrm{d} y^{\prime} .
$$

Limiting integration within the KL of three MFP, the impact from each is the most significant and in which $P_{c} y^{\prime} \ll 1$, the equation above is approximated as follows

$$
Z \cong \frac{1}{4} n v_{T} \int_{0}^{\frac{3}{P_{c}}} \frac{1}{y^{\prime}} \exp (-1) \mathrm{d} y^{\prime}=\left.\frac{1}{4} n v_{T} \exp (-1) \ln y^{\prime}\right|_{0} ^{\frac{3}{P_{c}}} \rightarrow \infty
$$

One can see that the integration above does not produce a meaningful value for the rate of collisions per unit area of the incompressible gas. We expect similar negative results of validation for the incompressible gas if we try other forms of MFP distribution functions, such as the power-law distribution function investigated by Dongari et al. [6] or Stops' exponential [13]. We support the claim 
in [15] that "[c]urrent claims of MFP variation normal to the nano-channel surfaces as a function of the confinement level are false".

\section{Physical Principles of the Ballistic Model in One-Dimensional Gas Space}

This section describes the BM's physical principles in one-dimensional incompressible steady-state gas flow at the uniform temperature, a lack of external field of the force, and low flow velocity (with the Mach number less than 0.3 ). These principles will be further applied to describe the steady-state gas flow in a micro-channel.

\subsection{Properties and Features of the Model Gas}

Here we assign the following properties of the model gas, which are adapted to the steady-state gas flow from the originally proposed in [25]:

1) The model gas enables a distant transport of one or more properties, including one or more of mass, momentum, and energy by particles being in a constant state of mostly random motion and interaction by collisions.

2) Each gas particle is assigned to travel by obeying a ballistic trajectory governed by a law of motion in free space. It overcomes a distance between any of the two points of the ballistic trajectory with a certain survival probability.

3) Each gas particle is adapted to transport a combination of one or more properties, comprising mass, momentum, and energy between a point of initial collision and a point of ending collision.

4) Each point within the space occupied by the model gas is treated as a point of collisions for converging particles, each following a ballistic trajectory with the same ending point simultaneously.

5) Each point of collisions is treated as either a point source for diverging ballistic particles or a point sink for converging ballistic particles.

6) Each of the particles moving from the point source to the point sink is treated as a property carrier. The property carrier is created in the point source by obtaining one or more properties of specific values being intrinsic to the model gas surrounding the point source. It is ended in the point sink by transferring one or more properties of specific values in the point sink.

7) The velocity of a point source equals the mass flow velocity of the model gas flow in a corresponding point of the initial collisions.

One can note from the above that the model gas properties differ from the properties typically assigned to the ideal gas.

\subsection{Properties and Features of the Gas-Solid Interface}

Here we assign the following additional unique properties to the model gas being in contact with a gas-solid interface revealing mixed diffuse and specular scattering of particles [4] [18]:

1) Each collision on a gas-solid interface of the model gas system, which has resulted in the scattering of the diffuse particles from the gas-solid interface, is 
treated as an act of interaction involving a property transport from the gas-solid interface to the scattered particle.

2) Each point of the diffuse particle scattering on the gas-solid interface is treated as a heterogeneous point source for each of the scattered particles.

3) The velocity of each of the heterogeneous point sources on the gas-solid interface equals the velocity of the gas-solid interface of corresponding points of diffuse particle scattering.

4) The point source strength of the heterogeneous point sources on the gas-solid interface is directly proportional to a property accommodation coefficient $\sigma$ in a corresponding point of diffuse particle.

Note: Diffuse scattering is an act of interaction involving property transfer from a gas-solid interface to a scattered particle. Specular scattering does not involve property exchange between the gas-solid interface and a scattered particle.

\subsection{Geometry of the Gas Flow and Ballistic Trajectories of Particles Involving in the Property Transport in a Micro-Channel}

Figure 3 illustrates the diversity of ballistic trajectories of particles involving in the property ( $\boldsymbol{u}_{x}$-momentum) transport in the one-dimensional configuration of a Newtonian model gas flow between infinite parallel plates spaced at a distance $H$. Ballistic trajectories 303 and 304 show the trajectories of particles from the control volume at $y^{\prime}$ into the control volume at $y$. Ballistic trajectories 305 and 306 show traces of ballistic particles, which are initiated from the preceding collisions in the gas space (such as the ballistic trajectories 303 and 304, respectively), into targeting point $y$.

For diffuse scattering with no adsorption effects after colliding with the surface, the particle flux per unit time diffusively scattered back from the gas-solid

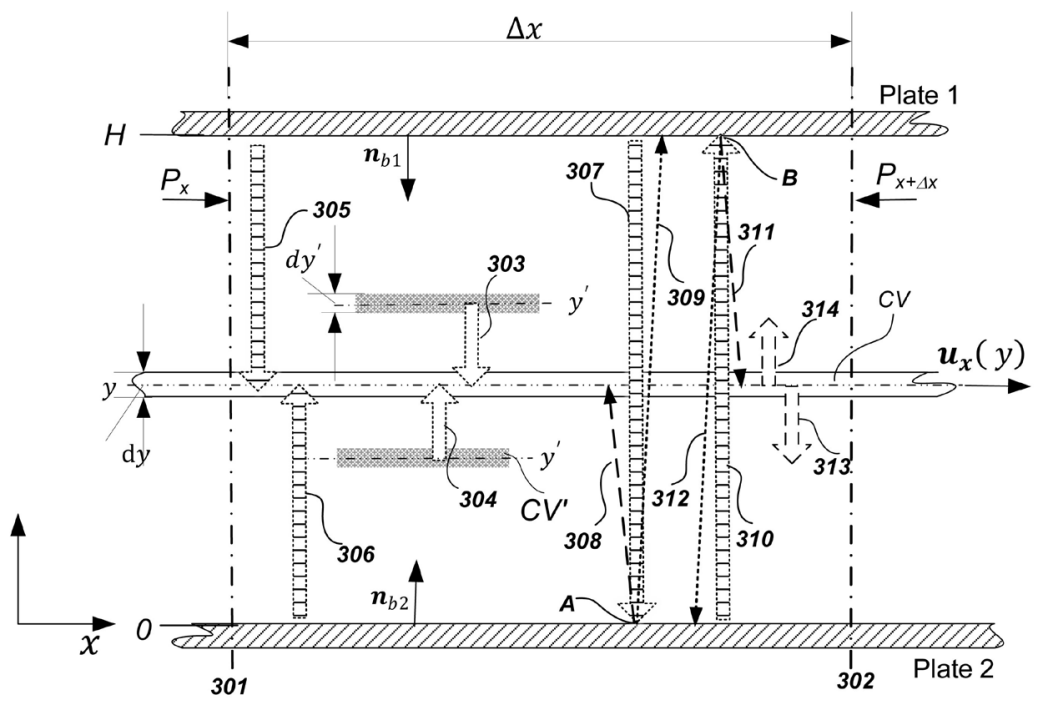

Figure 3. The schematic one-dimensional view of flow geometry and ballistic trajectories traces in micro-channel formed between two parallel plates. 
interface into the model gas system equals to the particle flux per unit time incident on the surface of the boundary with a negative sign. Here we have also accepted that particles diffusively scattered back from the gas-solid interface will accommodate a thermal velocity and other properties in a point of contact with the boundary's surface. Also, we recognize that only half of the particles near the scattering surface had a recent departure from the surface with the magnitude of velocity equal to the magnitude of thermal velocity corresponding to the gas-solid interface's temperature. Ballistic trajectories shown by arrow 307 indicate traces of ballistic particles initiated from the preceding collisions in the gas space from $y=H$ to $y=0$ and incident on targeting plate 2 at $y=0$ (point A). Ballistic trajectories 308 and 309 show traces of ballistic particles initiated from the diffuse scatterings on plate 2 but have a different destination. Particles following trajectory 308 have a chance to collide in the CV, while particles following trajectory 309 can travel by free path to the opposite plate 1. Analogously, ballistic trajectories shown by arrow 310 indicate traces of ballistic particles, which are initiated from the preceding collisions in the gas space from $y=0$ to $y$ $=\mathrm{H}$ and incident on targeting plate 1 at $y=H$ (point B). Ballistic trajectories 311 and 312 show traces of ballistic particles initiated from the diffuse scatterings on plate 1 (point B). Particles following trajectory 311 have a chance to collide in the $\mathrm{CV}$, while particles following trajectory 312 have a chance to travel by free path to the opposite plate 1. Ballistic trajectories shown by arrows 313 and 314 indicate the tracks of diverging ballistic particles, which are originated from the preceding collisions in the control volume surrounding point $y$.

The mentioned above trajectories were initially introduced in our publications [4] [18], and patent [25]. In this paper, we pay attention to a different group of trajectories such as 309 and 312, associated with the ballistic particles' bouncing between the being at rest confining plates, plate 1 and plate 2 . These particles are not participated in property exchange by collisions within the gas space.

\subsection{Principles of Construction of the Property Balance in Micro-Channel}

In the microscopic scale, the model gas flow is characterized by the group of particles of mass $m$, which move randomly and interact by collisions with effective collision cross-section $\sigma_{\mathcal{c}}$ In each of the points in space, the gas properties such as particle density $n$, the magnitude of thermal velocity $V_{T}$, and the vector of mass flow velocity $\boldsymbol{u}$ quantify the model gas. According to the BM, each point in space occupied by the model gas may serve as both a sink accumulating property delivered by converging ballistic particles from the entire model gas system and a source dispersing into the surrounding property by diverging ballistic particles. In the interests of simplicity, the particles are considered to have a unit mass unless otherwise stated.

Based on the BPPBS, we expect maintenance of an overall property balance in each collisions' points within the model gas system. In a steady-state gas flow, 
the BPPBS is formulated as follows: in each nonmoving point $y$, the net rate of property influx per unit volume, $\boldsymbol{B}_{i n}^{\Psi_{-} F S}$, formed the converging ballistic particles (each traveling along a ballistic trajectory with certain survival probability) from the model gas system is equated to the net rate of property efflux per unit volume, $\boldsymbol{B}_{\text {out }}^{\Psi}{ }^{F S}$, formed the diverging ballistic particles. This statement is expressed symbolically as

$$
\boldsymbol{B}_{\text {in }}^{\Psi_{-} F S}(y)=\boldsymbol{B}_{\text {out }}^{\Psi_{-} F S}(y)
$$

The table of the model parameters associated with defining the net rate of total property influx per unit volume and the net rate of total property efflux per unit volume is presented in Table 1 .

\subsection{Defining Components of a Net Rate of Total Property Influx Per Unit Volume in a Nonmoving Point of Gas Space in Micro-Channel}

Referring to Figure 3 and Table 1, we formulate the following equality for a micro-channel with gas-solid interfaces revealing diffuse particle scattering:

$$
\boldsymbol{B}_{i n}^{\Psi-F S}(y)=\boldsymbol{B}_{i n}^{\Psi-F}(y)+\boldsymbol{B}_{i n}^{\Psi+B D}(y)
$$

3.5.1. Formulating the Net Rate of Property Influx from the Surrounding Gas in the General Nonmoving Point $y$ in the Gas Space Confined between Two Parallel Plates

Modifying Equation (38) of [25] to applying to the one-dimensional steady-state gas system, we obtain:

$$
\boldsymbol{B}_{i n}^{\Psi-}{ }^{-}(y)=-\frac{1}{2} \frac{\partial}{\partial y} \int_{y_{b 2}}^{y_{b 1}} Q_{i}\left(y^{\prime}, y\right) Z_{V}\left(y^{\prime}\right) \frac{v\left(y^{\prime}, y\right)}{v_{T}\left(y^{\prime}\right)} \Psi\left(y^{\prime}\right) \mathrm{d} y^{\prime},
$$

where

$$
\begin{aligned}
Q_{i}\left(y^{\prime}, y\right) & =\exp \left(-P_{c}\left|\boldsymbol{y}-\boldsymbol{y}^{\prime}\right|\right), \\
Z_{V} & =\frac{1}{2} n P_{c} v_{T}, \\
\boldsymbol{v}\left(y^{\prime}, y\right) & =v_{T} \boldsymbol{n}_{i}+\boldsymbol{u}\left(y^{\prime}\right),
\end{aligned}
$$

and where $\boldsymbol{n}_{i}$ is a unit vector of arbitrary direction:

$$
\boldsymbol{n}_{i}=\frac{y-y^{\prime}}{\left|y-y^{\prime}\right|} .
$$

\subsubsection{Formulating the Net Rate of Property Influx from the Confining}

Plates Having Gas-Solid Interfaces with a Purely Diffuse Scattering of Particles in the General Nonmoving Point $y$ in the Gas Space Confined between Two Parallel Plates Being at Rest

Modifying Equations (40), (41), (49), and (50) of [25] to applying to a one-dimensional steady-state gas system, we obtain:

$$
\boldsymbol{B}_{i n}^{\Psi-B D 1}(y)=Z_{b} \frac{\partial}{\partial y} Q_{i b 1}(y, H) \Psi_{b 1},
$$


Table 1. List of the model parameters associated with defining the net rate of total property influx per unit volume and the net rate of total property efflux per unit volume.

\begin{tabular}{|c|c|}
\hline Parameters & Short description \\
\hline$\frac{\partial}{\partial y}$ & the divergence operator in a one-dimensional configuration \\
\hline$y$ & position of the ending point of the converging particle \\
\hline$y^{\prime}$ & position of the starting point of the converging particle \\
\hline$u(y)$ & mass flow velocity in the point $y$ \\
\hline$v_{T}(y)$ & the average magnitude of the thermal velocity of converging particle in point \\
\hline$Z_{V}\left(y^{\prime}\right)$ & the rate of collisions per unit volume at the point of the collision $y^{\prime}$ \\
\hline $\overrightarrow{\boldsymbol{v}}(y)$ & velocity vector in the ending point $y$ \\
\hline$Q_{i}\left(y^{\prime}, y\right)$ & $\begin{array}{l}\text { the probability of free path traveling along the ballistic trajectory of the } \\
\text { converging ballistic trajectory starting at } y^{\prime} \text { and ending } y\end{array}$ \\
\hline$\Psi_{\text {out }}(y)$ & property content of a diverging point $y$ particle \\
\hline$\Psi_{i n}\left(y^{\prime}\right)$ & property content of a diverging point $y^{\prime}$ particle and heading to $y$ \\
\hline$n$ & incompressible gas particles density \\
\hline$n_{e f}\left(y^{\prime}\right)$ & $\begin{array}{l}\text { effective gas-particle density in point } y^{\prime} \text { associated with particles carrying } \\
\text { transported property }\end{array}$ \\
\hline$m$ & particle mass \\
\hline$\sigma_{c}$ & the cross-section of collisions \\
\hline$P_{c}=\sigma_{c} n$ & the number of particles placed within a collision tube of a unit length \\
\hline$y_{b 1}$ & a position of the upper plate of the micro-channel \\
\hline$y_{b 2}$ & a position of the lower plate of the micro-channel \\
\hline$V$ & the volume of integration over space occupied by the model gas \\
\hline $\boldsymbol{v}_{+}\left(y, y^{\prime}\right)$ & the velocity vector of the diverging particle in point $y^{\prime}$ \\
\hline$Q_{+}\left(y, y^{\prime}\right)$ & the probability of free path traveling along the ballistic trajectory from $y$ to $y^{\prime}$ \\
\hline $\boldsymbol{B}_{i n}^{\Psi_{i n} F S}(y)$ & the net rate of property influx per unit volume from the model gas system \\
\hline $\boldsymbol{B}_{i n}^{\Psi_{-} F}(y)$ & $\begin{array}{l}\text { the net rate of property influx per unit volume from the surrounding gas } \\
\text { (trajectories } 303 \text { and 304) }\end{array}$ \\
\hline $\boldsymbol{B}_{i n}^{\Psi_{i n} B D}(y)$ & $\begin{array}{l}\text { the net rate of property influx per unit volume from the boundaries } \\
\text { (trajectories } 315 \text { and } 316 \text {, diffuse component) }\end{array}$ \\
\hline$Q_{21}=\exp \left(-P_{c} H\right)$ & $\begin{array}{l}\text { the probability of free path traveling along the ballistic trajectory between } \\
\text { confining plates in incompressible gas }\end{array}$ \\
\hline
\end{tabular}

and

$$
\boldsymbol{B}_{i n}^{\Psi-B D 2}(y)=-Z_{b} \frac{\partial}{\partial y} Q_{i b 2}(y, 0) \Psi_{b 2},
$$


where $\boldsymbol{B}_{i n}^{\Psi-B D 1}(y)$ is the net rate of property influx per unit volume from the boundary at $y_{b 1}=H \quad$ (trajectory 308) and $\boldsymbol{B}_{i n}^{\Psi-B D 2}(y)$ is the net rate of property influx per unit volume from the boundary at $y_{b 2}=0$ (trajectory 307), and $Z_{b}$ is the rate of collisions per unit area on plate 1 or plate 2 . In the equations above, $Q_{i b 1}(y, H)$ is the survival probability that a particle will have traveled along the ballistic trajectory in incompressible gas at the uniform temperature from $y_{b 1}=H$ to $y$

$$
Q_{i b 1}(y, H)=\exp \left(-P_{c}(H-y)\right)
$$

$Q_{i b 2}(y, 0)$ is the survival probability that a particle will have traveled along the ballistic trajectory in incompressible gas at the uniform temperature from $y_{b 1}=0$ to $y$

$$
Q_{i b 2}(y, 0)=\exp \left(-P_{c} y\right)
$$

and $\Psi_{b 1}$ and $\Psi_{b 2}$ are property values acquired by diffusively scattered particles from plate 1 and plate 2 , respectively.

\subsubsection{Formulating a Net Rate of Total Property Efflux Per Unit Volume} from the General Nonmoving Point of Gas Space in Micro-Channel

We have recognized that the linear dimensions of the main control volume surrounding point $y$ need to be sufficiently small to prevent two and more consecutive collisions of the same particle with other particles within the main control volume CV [4]. In Figure 3, the arrows 313 and 314 ballistic indicate the trajectory of gas particles escaping the $\mathrm{CV}$ positioned in point $y$.

Modifying Equation (73) of [25] to applying to the one-dimensional steady-state gas system, we obtain:

$$
\boldsymbol{B}_{\text {out }}^{\Psi-F S}(y)=\frac{1}{2} n\left\{\frac{\partial}{\partial y}\left[Q_{+}\left(y, y^{\prime}\right) \boldsymbol{v}_{+}\left(y, y^{\prime}\right) \Psi_{\text {out }}(y)\right]\right\}_{y^{\prime} \rightarrow y},
$$

where

$$
Q_{+}\left(y, y^{\prime}\right)=\exp \left(-P_{c}\left|y^{\prime}-y\right|\right)
$$

for incompressible gas.,

$$
\boldsymbol{v}_{+}\left(\boldsymbol{y}, \boldsymbol{y}^{\prime}\right)=v_{T} \boldsymbol{n}_{+}+\boldsymbol{u}(y)
$$

and where $\boldsymbol{n}_{+}$is a unit vector of arbitrary direction:

$$
\boldsymbol{n}_{i}=\frac{y^{\prime}-y}{\left|y^{\prime}-y\right|} \text {. }
$$

\section{Analytical Representation of a General Integral Form of the Property Balance Equation in the Micro-Channel}

The integro-differential form of property balance equation is formulated by Equation (41) given below, which is obtained by substitution of Equations (28), (33), (34), and (37) in Equation (26): 


$$
\begin{aligned}
& -\frac{1}{2} \frac{\partial}{\partial y} \int_{0}^{H} Q_{i}\left(y^{\prime}, y\right) Z_{V}\left(y^{\prime}\right) \frac{v\left(y^{\prime}, y\right)}{v_{T}\left(y^{\prime}\right)} \Psi_{i n}\left(y^{\prime}\right) \mathrm{d} y^{\prime} \\
& +Z_{b} \frac{\partial}{\partial y} Q_{i b 1}(y, H) \Psi_{b 1}-Z_{b} \frac{\partial}{\partial y} Q_{i b 2}(y, 0) \Psi_{b 2}+\dot{Q}_{\Psi} \\
& =\frac{1}{2} n\left\{\frac{\partial}{\partial y}\left[Q_{+}\left(y, y^{\prime}\right) \boldsymbol{v}_{+}\left(y, y^{\prime}\right) \Psi_{o u t}(y)\right]\right\}_{y^{\prime} \rightarrow y}
\end{aligned}
$$

where $\dot{Q}_{\Psi}$ is a term representing a generation of a transported quantity within the model gas system, resulting from transmitting property across the surfaces of inlet and outlet openings. Suppose the property transport is the momentum transport. In that case, the generation term in a confined model gas system results from forces acting on the model gas in the form of the external pressure force, $P$, applied across the surface bounding the system. More specifically, normal gradient pressure force acting on the model gas and prompting model gas flow represents the effect of surroundings on the momentum change. Here point $y$ is not included in integration for converging ballistic particles. Substituting Equations (29), (30), (31), (35), (36), (38), and (39) in the equation above, and executing differentiation, we obtain the following general integral form of the property balance equation:

$$
\begin{aligned}
& \frac{1}{2} Z_{V} P_{c} \int_{0}^{H} \exp \left(-P_{c}\left|\boldsymbol{y}-\boldsymbol{y}^{\prime}\right|\right) \boldsymbol{\Psi}_{\text {in }}\left(y^{\prime}\right) \mathrm{d} y^{\prime} \\
& +\frac{1}{2} Z_{V} P_{c} \int_{0}^{H} \exp \left(-P_{c}\left|\boldsymbol{y}-\boldsymbol{y}^{\prime}\right|\right) \boldsymbol{n}_{i} \frac{u\left(y^{\prime}\right)}{v_{T}} \Psi_{\text {in }}\left(y^{\prime}\right) \mathrm{d} y^{\prime} \\
& +Z_{b} P_{c} \exp \left(-P_{c}(H-y)\right) \Psi_{b 1}+Z_{b} P_{c} \exp \left(-P_{c} y\right) \Psi_{b 2}+\dot{Q}_{\Psi} \\
& =\frac{1}{2} n\left\{\frac{\partial}{\partial y}\left[\exp \left(-P_{c}\left|y^{\prime}-y\right|\right)\left(v_{T} \boldsymbol{n}_{+}+\boldsymbol{u}(y)\right) \Psi_{\text {out }}(y)\right]\right\}_{y^{\prime} \rightarrow y}
\end{aligned}
$$

\subsection{Analytical Representation of a General Integro-Differential Form of Mass Balance Equation in the Micro-Channel}

To formulate a general integro-differential form of mass balance equation in each nonmoving point of space occupied by the gas, we modify Equation (42) by assigning

$$
\Psi_{\text {in }}=\Psi_{\text {out }}=\Psi_{b 1}=\Psi_{b 2}=1, \dot{Q}_{\Psi}=0
$$

executing differentiation in the right-hand of the resulting equation followed by executing limit $\boldsymbol{y}^{\prime} \rightarrow y$, and rearranging the terms. Then, we obtain the following general integro-differential form of the mass balance equation

$$
\begin{aligned}
Z_{V}+\frac{1}{2} n \frac{\partial}{\partial y} \boldsymbol{u}= & \frac{1}{2} Z_{V} P_{c} \int_{0}^{H} \exp \left(-P_{c}\left|\boldsymbol{y}-\boldsymbol{y}^{\prime}\right|\right) \mathrm{d} y^{\prime} \\
& +\frac{1}{2} Z_{V} P_{c} \int_{0}^{H} \exp \left(-P_{c}\left|y-y^{\prime}\right|\right) \boldsymbol{n}_{i} \frac{\boldsymbol{u}\left(y^{\prime}\right)}{v_{T}} \mathrm{~d} y^{\prime} \\
& +Z_{b} P_{c} \exp \left(-P_{c}(H-y)\right)+Z_{b} P_{c} \exp \left(-P_{c} y\right)
\end{aligned}
$$




\subsection{Analytical Representation of a General Integro-Differential Form of Momentum Balance Equation in the Micro-Channel}

To formulate a general integro-differential form of momentum (along with $y$-axis) balance equation in each nonmoving point of space occupied by the gas, we modify Equation (42) by assigning

$$
\Psi_{\text {in }}=v_{T} \boldsymbol{n}_{i}+\boldsymbol{u}\left(y^{\prime}\right) ; \Psi_{\text {out }}=v_{T} \boldsymbol{n}_{+}+\boldsymbol{u}(\boldsymbol{y}) ; \Psi_{b 1}=-v_{T} ; \Psi_{b 2}=+v_{T} ; \dot{Q}_{\Psi}=0
$$

executing differentiation in the right-hand of the resulting equation followed by executing limit $\boldsymbol{y}^{\prime} \rightarrow y$, and rearranging the terms. Then, we obtain the following general integro-differential form of the momentum balance equation

$$
\begin{aligned}
2 Z_{V} u+\frac{1}{2} n \frac{\partial}{\partial y} u^{2}= & \frac{1}{2} Z_{V} P_{c} v_{T} \int_{0}^{H} \exp \left(-P_{c}\left|y-y^{\prime}\right|\right) \boldsymbol{n}_{i} \mathrm{~d} y^{\prime} \\
& +Z_{V} P_{c} \int_{0}^{H} \exp \left(-P_{c}\left|y-y^{\prime}\right|\right) \boldsymbol{u}\left(y^{\prime}\right) \mathrm{d} y^{\prime} \\
& +\frac{1}{2} Z_{V} P_{c} \frac{1}{v_{T}} \int_{0}^{H} \exp \left(-P_{c}\left|y-y^{\prime}\right|\right) \boldsymbol{u}^{2} \boldsymbol{n}_{i} \mathrm{~d} y^{\prime} \\
& -Z_{b} P_{c} v_{T} \exp \left(-P_{c}(H-y)\right)+Z_{b} P_{c} v_{T} \exp \left(-P_{c} y\right)
\end{aligned}
$$

\subsection{Solving a System of Mass-Balance and Momentum Balance Equations for Determining $Z_{b}$}

According to the BPPBS, the mass-balance and momentum-balance shall be maintained at every point of the gas space [4]. Recognizing that in both Equation (44) and Equation (46), there are two independent variables, $\boldsymbol{u}$ and $Z_{b}$, one can solve a system of the above-mentioned equations and obtain a solution that is $\boldsymbol{u}=0$ and

$$
Z_{b}=\frac{Z_{V}}{2 P_{c}}=n \frac{v_{T}}{4} .
$$

The fact that the result of the derivation of $Z_{b}$ from the mass- and momentum-balance equations is identical to the result of the derivation of the rate of collisions per unit area of an ideal gas according to the kinetic theory of gases supports the Ballistic Model's validity.

The functional relationship expressed by the equation above may also be obtained by analyzing fluxes formed by the ballistically traveling particles. Referring to Figure 3 and Equations (45) and (47) of [25] and considering symmetricity because of the identity of properties of gas-solid interfaces of both plate 1 and plate 2, we may write the following relationship:

$$
\boldsymbol{J}_{F S}^{1}=-\boldsymbol{J}_{F S}^{2}=\boldsymbol{N}_{21}^{1}+\boldsymbol{N}_{2}^{1},
$$

where $\boldsymbol{J}_{F S}^{1}$ and $\boldsymbol{J}_{F S}^{2}$ are the particle fluxes per unit time on the gas-solid interfaces of plate 1 and plate 2, respectively, $N_{21}^{1}$ is the particle flux per unit time on the gas-solid interface of plate 1 , which is associated with the incident on the gas-solid interface of ballistic particles from the gas space following ballistic trajectories 307, and $N_{2}^{1}$ is the particle flux per unit time on the gas-solid interface of plate 1, which is associated with an incident on the gas-solid interface of 
ballistic particles from originated from diffuse scattering on the gas-surface interface of plate 4. Using Equations (43) and (42) of [25] as templates, we determine

$$
N_{21}^{1}=\frac{1}{2} \int_{0}^{H} Q_{i}\left(y^{\prime}, y\right) Z_{V} \mathrm{~d} y^{\prime}=\frac{1}{2 P_{c}} Z_{V}\left[1-\exp \left(-P_{c} H\right)\right]
$$

and

$$
\boldsymbol{N}_{2}^{1}=Z_{b} \exp \left(-P_{c} H\right),
$$

respectively. Referring again to Figure 3 and Equations (46) and (48) of [25], we formulate the following relationship:

$$
Z_{b}=-\boldsymbol{J}_{F S}^{1} \boldsymbol{n}_{b 1}=-\left(\boldsymbol{N}_{21}^{1}+\boldsymbol{N}_{2}^{1}\right) \boldsymbol{n}_{b 1}=-\boldsymbol{J}_{F S}^{2} \boldsymbol{n}_{b 2} .
$$

Substituting Equations (48)-(50) in Equation (51) and taking $\boldsymbol{n}_{b 1}=-1$ and performing algebraic operations, we finally obtain

$$
Z_{b}=\frac{Z_{V}}{2 P_{c}}=n \frac{v_{T}}{4},
$$

which is identical to Equation (47). The above implies that $Z_{b}$ is constant at any Knudsen number.

\subsection{Finding the Effect of Rarefication on the Effective Density of Particles in the Gas Space, Which Can Carry and Exchange by Collisions $\boldsymbol{u}_{\boldsymbol{x}}$-Momentum}

Here we shall admit that flux $N_{2}^{1}$ produces an impact on the rate of collisions on the gas-solid interface, but it does not directly involve the collisional property exchange within the gas volume. Considering the above, the effective rate of collision per unit area, which is involved directly in the collisional property exchange within the gas volume, is defined from the equation given below

$$
Z_{b}^{e f}=Z_{b}+N_{2}^{1} n_{b 1}=Z_{b}\left(1-\exp \left(-P_{c} H\right)\right) .
$$

Referring to Figure 3, one can note that only gas particles in the space between the plates following converging trajectories 305 and 306, diffuse particles from the plates following converging trajectories 308 and 311, and ballistic gas particles escaping the $\mathrm{CV}$ positioned in point $y$ following diverging trajectories 313 and 314 participate in the $\boldsymbol{u}_{x}$ momentum transport. This group of active gas particles and associated with it the effective rate of collision per unit volume, $Z_{V}^{e f}$, in the CV at $y$ is evaluated from Equation (44), in which $\boldsymbol{u}=0, Z_{V}$ is substituted by $Z_{V}^{e f}$, and $Z_{b}$ is substituted by $Z_{b}^{e f}$ :

$$
\begin{aligned}
Z_{V}^{e f}(y)= & \frac{1}{2} P_{c} \int_{0}^{H} Z_{V}^{e f}\left(y^{\prime}\right) \exp \left(-P_{c}\left|\boldsymbol{y}-\boldsymbol{y}^{\prime}\right|\right) \mathrm{d} y^{\prime} \\
& +Z_{b}^{e f} P_{c} \exp \left(-P_{c}(H-y)\right)+Z_{b}^{e f} P_{c} \exp \left(-P_{c} y\right)
\end{aligned}
$$

Resolving the equation above with respect to $Z_{V}^{e f}$, we obtain:

$$
Z_{V}^{e f}=2 P_{c} Z_{b}^{e f}=Z_{V}\left(1-\exp \left(-P_{c} H\right)\right) .
$$

Recognizing that the rate of collisions per unit length, $P_{\mathcal{o}}$ is constant for in- 
compressible gas, we may introduce the effective density of particles currying $\boldsymbol{u}_{x}$ momentum, $n_{\text {eff }}$ by expressing $Z_{V}^{\text {eff }}$ as

$$
Z_{V}^{e f f}=\frac{1}{2} P_{c} n_{e f f} \sigma_{c} v_{T} .
$$

Now substituting Equations (30), (55), and (56) in Equation (54) and executing integration and algebra operations and replacing $P_{c} H$ by $\mathrm{Kn}^{-1}$, we may define the normalized effective density, $k_{e f}$ as follows:

$$
k_{e f}=\frac{n_{e f}}{n}=\frac{Z_{V}^{e f}}{Z_{V}}=1-\exp \left(-\frac{1}{K n}\right),
$$

where $K n$ is the Knudsen number defined as the ratio of the mean free path $\lambda_{f}=1 / P_{c}$ and the representative length scale $H$. Results for the normalized effective density profile between two parallel plates are presented in Figure 4.

We intentionally provided a graphical interpretation of Equation (54) to highlight that the normalized effective density is constant within the gas space and depends only on the Knudsen number. As the Knudsen number's value increases from 0.2 to 20 , the effective normalized density decreases from 1 to 0.048 .

\section{Influence of the Effective Density of $u_{x}$-Momentum Carriers on the Rarefied Gas Flow in the Microchannel}

Considering the effect of the confining plates on the density of $\boldsymbol{u}_{\boldsymbol{x}}$-momentum carriers in the channel's gas space, we analyze the velocity profile generated in the model gas due to the pressure gradient along the channel. We use a similar approach that has been initially introduced in our publications [4] and [18].

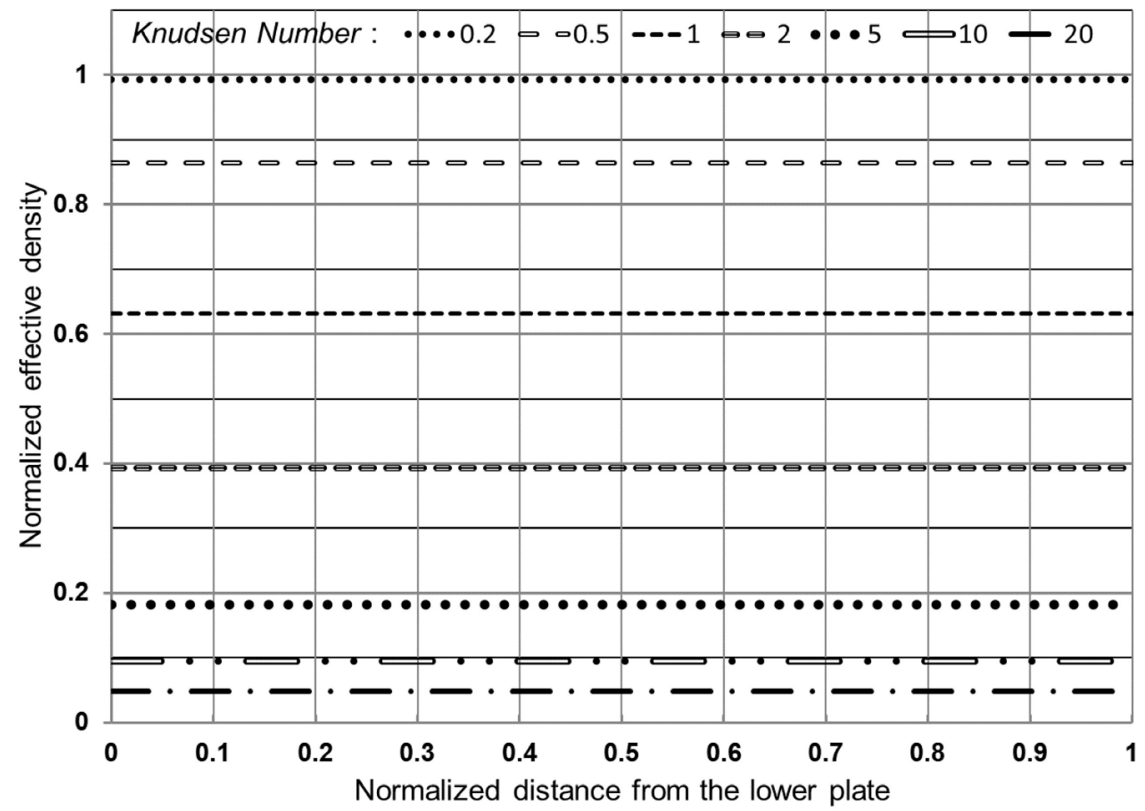

Figure 4. Normalized effective density profile in the microchannel between two infinite parallel plates. 
Concerning Figure 3, the gas flow, with the velocity distribution $\boldsymbol{u}_{x}(y)$, is forced by the pressure gradient $-\frac{P_{x+\Delta x}-P_{x}}{\Delta x}=-\frac{\mathrm{d} P}{\mathrm{~d} x}$. The main control volume (CV) of the unit length of volume $\mathrm{d} V \stackrel{\Delta x}{=} \Delta x \Delta y$ is at $y$ within the model gas flow confined by two being at rest parallel plates at $y=0$ and $y=H$. Plate 1 and Plate 2 confine across the $y$-axis a model gas flow along the $x$-axis and surfaces 301 and 302 positioned at distance $\Delta x$ bound a portion of the model gas system along the $\mathrm{x}$-axis. Here we assume purely diffuse scattering of the particles from the walls $\sigma=1$, so that the probability, for an incident particle, to accommodate momentum from the gas-solid interface and to scatter back in the model gas as a diffuse particle equals to one. Also, because of the zero-velocity of the confining plates, the particles scattered from the walls do not carry $\boldsymbol{u}_{x}$-momentum, so that the fluxes associated with trajectories 308 and 311 are zeroed. Here we also consider that the magnitude of the thermal velocity of the particles is much higher than the magnitude of the mass flow velocity vector along the $\mathrm{x}$-direction, $\left|\boldsymbol{u}_{x}\right| \ll v_{T}$. To formulate a general integro-differential form of $\boldsymbol{u}_{x}$-momentum balance equation in each nonmoving point of space occupied by the gas, we modify Equation (42) by assigning

$$
\Psi_{\text {in }}=m \boldsymbol{u}_{x}\left(y^{\prime}\right) ; \Psi_{\text {out }}=m \boldsymbol{u}_{x}(y) ; \Psi_{b 1}=0 ; \Psi_{b 2}=0 ; \dot{Q}_{\Psi}=-\frac{\mathrm{d} P}{\mathrm{~d} x}
$$

executing differentiation in the right-hand of the resulting equation followed by executing limit $\boldsymbol{y}^{\prime} \rightarrow y$, and rearranging the terms. Then, we obtain the following general integro-differential form of the momentum balance equation:

$$
m Z_{V} \boldsymbol{u}_{x}=-k_{e f} \frac{\mathrm{d} P}{\mathrm{~d} x}+\frac{1}{2} P_{c} m Z_{V}^{e f} \int_{0}^{H} \exp \left(-P_{c}\left|y-y^{\prime}\right|\right) \boldsymbol{u}_{x}\left(y^{\prime}\right) \mathrm{d} y^{\prime}
$$

One can note that the equation above, in which $k_{\text {eff }}=1$ and $Z_{V}^{e f}=Z_{V}$, is identical to Equation (112) of our paper [4], in which $\sigma=1$. We will explain usage of $k_{e f}$ upon the following analysis. We have recognized that some of the particles initiated from collisions in the gas space and carrying $\boldsymbol{u}_{x}$ momentum may travel by free path to a wall, then after accommodation with the gas-solid interface of a wall diffusively scatter back in the gas space. Here we shall recite that "the cause of the thermalization and "diffuse" escape of particles from the surface lies not in the trapping of molecules by the surface, as assumed by Maxwell himself and repeated in practically all the literature published on this question, but in the relaxation, in interaction with the phonon subsystem" [26]. Since the phonon-relaxation time is about $10^{-11}-10^{-10} \mathrm{sec}$, the thermalized particle scatters back from a gas-solid interface almost immediately. All the particles scattered from the walls do not carry $\boldsymbol{u}_{x}$-momentum. Some of these scattered particles may even travel to the opposite wall by free path. Such group of the particles will not participate in $\boldsymbol{u}_{x}$ momentum transport anymore. This effect was not taken into consideration in our publications [4] and [18].

Here we also understand that the flow through the channel is motivated by the surface force exerted by the surroundings on the CV through the pressure force, and the pressure is directly proportional to gas particles density. We suggest 
that, in the gas of particle density $n$ and the effective density $n_{\text {eff }}$ the effective pressure force applied to the differential $\mathrm{CV}$ at $y$ through the active gas particles may be expressed as

$$
F_{s}=-k_{e f} \frac{\mathrm{d} P}{\mathrm{~d} x}=-\frac{\mathrm{d} P}{\mathrm{~d} x}\left[1-\exp \left(-P_{c} H\right)\right]
$$

Here we need to admit that while evaluating the effective pressure force applied to the differential CV, we used the effective density $n_{e f}$ of active particles, which is represented in the right-hand terms of Equation (59) by the coefficient $k_{e f}$ However, in the left-hand term and the second right-hand term of Equation (59), we shall use the density of the incompressible gas, $n$. Such differentiation may be explained by the fact that eventually, all particles of the incompressible gas system are needed to satisfy the laws of mass balance and momentum balance (see the system of Equations (44) and (46) and Equation (47) as the solution of the system). As such, all particles of the incompressible gas system are involved in $\boldsymbol{u}_{x}$ momentum balancing in each point of the gas space according to Equation (59). Substituting $k_{e f}$ taken from the equation above and Equation (30) in Equation (59), we finally obtain:

$$
\begin{aligned}
\boldsymbol{u}_{x}= & -\frac{2 H}{n P_{c} H v_{T}} \frac{1}{m} \frac{\mathrm{d} P}{\mathrm{~d} x}\left[1-\exp \left(-P_{c} H\right)\right] \\
& +\frac{1}{2} P_{c} \int_{0}^{y} \exp \left(-P_{c}\left(y-y^{\prime}\right)\right) \boldsymbol{u}_{x}\left(y^{\prime}\right) \mathrm{d} y^{\prime} \\
& +\frac{1}{2} P_{c}\left[1-\exp \left(-P_{c} H\right)\right] \int_{y}^{H} \exp \left(-P_{c}\left(y^{\prime}-y\right)\right) \boldsymbol{u}_{x}\left(y^{\prime}\right) \mathrm{d} y^{\prime}
\end{aligned}
$$

Normalizing Equation (61) to

$$
u_{\max }=\left(-\frac{H}{m n v_{T}} \frac{\mathrm{d} P}{\mathrm{~d} x}\right)
$$

and assigning

$$
\boldsymbol{U}_{x}(y)=\frac{\boldsymbol{u}_{x}(y)}{u_{\max }}
$$

we obtain:

$$
\begin{aligned}
\boldsymbol{U}_{x}(y)= & \frac{2}{P_{c} H}\left[1-\exp \left(-P_{c} H\right)\right] \\
& +\frac{1}{2} P_{c} \exp \left(-P_{c} y\right)\left[1-\exp \left(-P_{c} H\right)\right] \int_{0}^{y} \exp \left(P_{c} y^{\prime}\right) \boldsymbol{U}_{x}\left(y^{\prime}\right) \mathrm{d} y^{\prime} \\
& +\frac{1}{2} P_{c} \exp \left(P_{c} y\right)\left[1-\exp \left(-P_{c} H\right)\right] \int_{y}^{H} \exp \left(-P_{c} y^{\prime}\right) \boldsymbol{U}_{x}\left(y^{\prime}\right) \mathrm{d} y^{\prime}
\end{aligned}
$$

where $\boldsymbol{U}_{x}(y)=\frac{\boldsymbol{u}_{x}(y)}{u_{\max }}$ is a non-dimensional velocity of the gas flow in the channel. The equation above's explicit solution is obtained numerically by sequential approximation described previously in [18].

Further integration of $\boldsymbol{U}_{x}(y)$ along $y$-direction and normalization by $H$ will result in finding a normalized volume flow rate $U_{x A}$ : 


$$
U_{x A}=\frac{1}{H} \int_{0}^{H} \boldsymbol{U}_{x}(y) \mathrm{d} y .
$$

Then we can obtain the normalized velocity profile as:

$$
\boldsymbol{U}_{x N}(y)=\frac{\boldsymbol{U}_{x}(y)}{U_{x A}}=\frac{\boldsymbol{U}_{x}(y)}{\frac{1}{H} \int_{0}^{H} \boldsymbol{U}_{x}(y) \mathrm{d} y} .
$$

In Figure 5, we show the velocity profiles $\boldsymbol{U}_{x N}(y)$ in the micro channel between two infinite parallel plates at different Knudsen numbers. We observe in Figure 5 that the velocity is nonzero at the channel walls, even at $K n=0.001$. The slip velocity at the walls is increased from 0.006 at $K n=0.001$ to 0.988 at $K n=5$. Also, one may note that the normalized flow velocity is almost constant across the channel at $K n>5$.

Figure 6 depicts a comparison of the normalized volume flow rate, $U_{x A}$, obtained from Equation versus inverse Knudsen number, $\delta=\sqrt{\pi} /(2 K n)$ through the microchannel obtained from the present theory against the analysis of Guo et al. [3], Kanki and Iuchi [27], obtained from [28], and the experimental data of Dong for " $\mathrm{H}_{2}, \mathrm{He}, \mathrm{CO}_{2}$, freon 12, and air", obtained from [3]. One can see that data of the present theory agree well with the experimental data in the entire range of the inverse Knudsen number (correlation factor in the range of $0.01 \leq \delta \leq 40$ is about 0.995 ). While BGK model with the boundary condition of diffuse reflection of Kanki and Iuchi [27] is in a good correlation with Dong's experimental data in the range of $0.2 \leq \delta \leq 40$, it shows a large deviation in the range of $\delta<0.2$. One may note a good agreement between the ENSC presented in [3] and the Dong's experimental data. However, as we discussed above, we are doubtful about the validity of the artificial incorporation in the Navier-Stokes equations of "the geometry-dependent viscosity and the $\lambda$-dependent heuristic boundary conditions [3]".

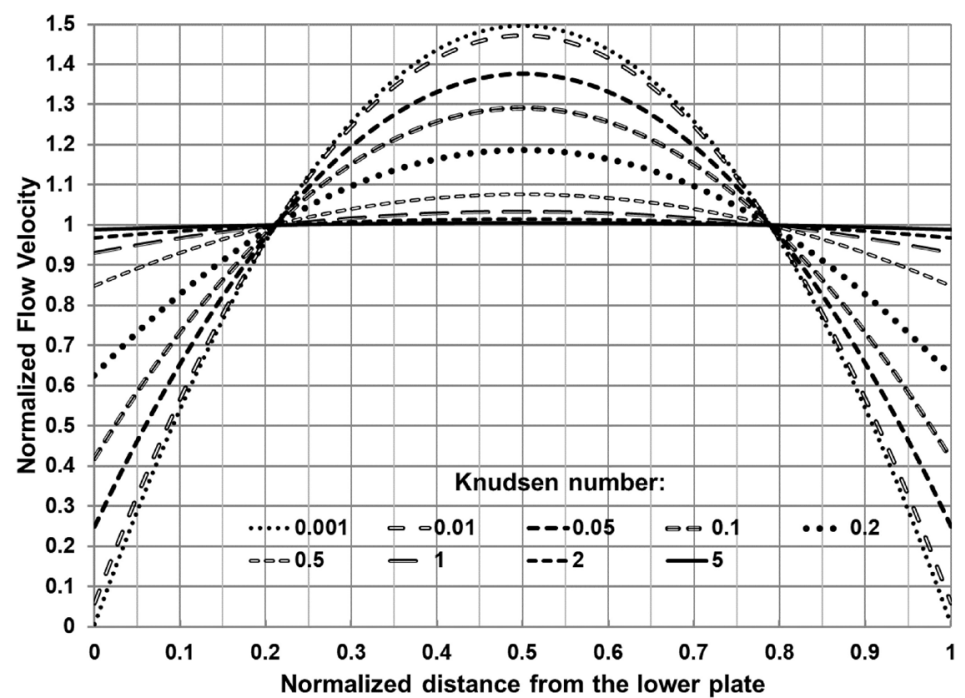

Figure 5. Normalized flow velocity profiles in the microchannel between two infinite parallel plates. 


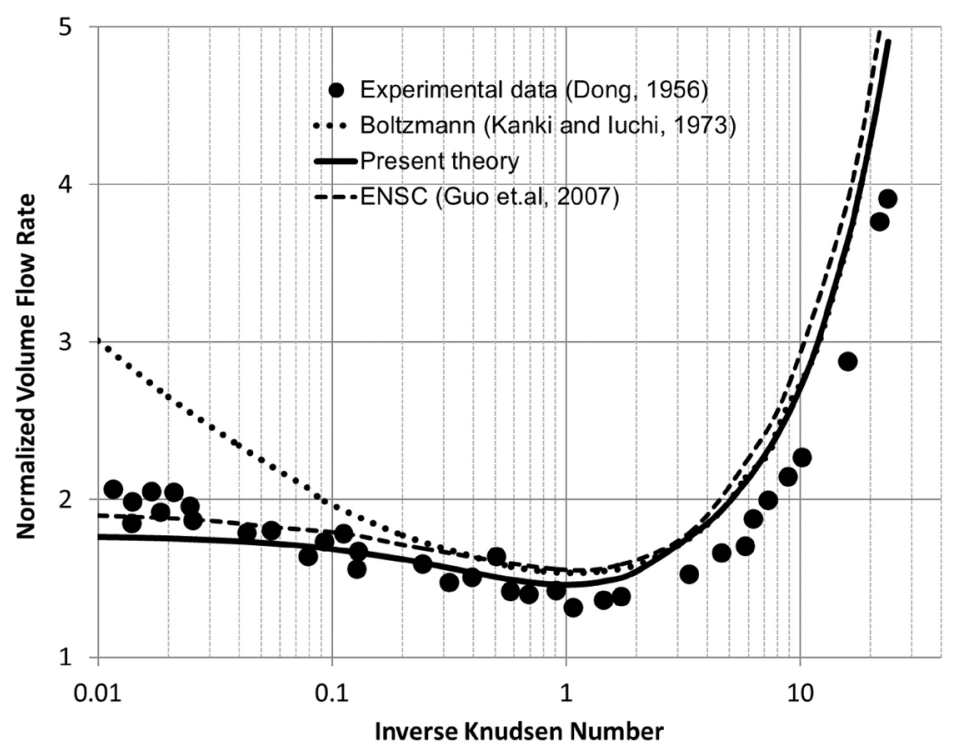

Figure 6. Variation of the normalized volume flow rate $U_{x A}$ as a function of the inverse Knudsen number $\delta$ according to the current theory and comparison with the Boltzmann equation [27], experimental data [3] (Dong, 1956), and ENSC [3].

It is also worth noting that the normalized volume flow rate $U_{x A}$ as a function of the inverse Knudsen number, according to the current theory, has the so-called "Knudsen minimum" at $\delta \cong 0.9$. It is in good agreement with Knudsen's observation of the minimum at about $K n \sim 1$ [29].

\section{Deriving a Differential form $u_{x}$ Momentum Balance Equation Governing Gas Flow in the Channel between Two Parallel Plates Due to the Pressure Gradient along the Channel and Its Analysis}

We have simulated gas flow in a plane channel using the BPPBS and obtained the gas flow velocity profile in the implicit form shown by Equation (64). It would also be interesting to obtain and analyze a differential form of the equation governing such gas flow in the channel. Below we show a way to obtaining a differential form $u_{x}$ momentum balance equation governing gas flow in the channel between two parallel plates due to the pressure gradient along the channel, like the presented in Step 2 of Section 4.4.3 of our paper [4].

Applying to Equation (64) the method of differentiation for integral equations (ones, twice, and so on) with subsequent elimination of the terms belonging to the original equation [30], we derived the following differential equation with respect to the independent variable $U_{x}(y)$ :

$$
\frac{1}{P_{c}^{2}} \frac{\mathrm{d}^{2}}{\mathrm{~d} y^{2}} U_{x}(y)=U_{x}(y) \exp \left(-\frac{1}{K n}\right)-2 K n\left[1-\exp \left(-\frac{1}{K n}\right)\right] .
$$

The equation above is different from the classical Navier-Stokes equation applicable to incompressible Newtonian flow. Here we will not further proceed to obtain an explicit analytical solution of the differential equation above, as we did 
in Section 4.4.3 of our paper [4]. However, we point out that Equation (61) is an implicit solution of the differential equation above, which can be easily verified by substitution of Equation (61) in the equation above and the execution of differentiation and algebra operations. One can also note that for $K n \ll 1$, Equation (67) is reduced to

$$
\frac{1}{P_{c}^{2}} \frac{\mathrm{d}^{2}}{\mathrm{~d} y^{2}} U_{x}(y)=-2 K n
$$

which, if it is multiplied by $u_{\max }$, is identical to Equation (104) of [4] having an analytical solution expressed by Equation (109) of [4]. However, for $K n \gg 1$, Equation (67) is roughly reduced to

$$
U_{x}(y) \cong 2 .
$$

Here we consider the collision-dominated flow regime, in which the relative change of any property value (velocity along the channel) is insignificant on the length scale of the average distance between the model gas particles, $\frac{1}{P_{c}}$ (see Equation (61) of [4]). Therefore, in this approximation, the left-hand term in Equation (67) is neglected. One can note that the identical result can be obtained from Equation (64) for $\frac{1}{P H}=K n \gg 1$, indicating that, the velocity of gas flow is constant across the channel and is independent of the Knudsen number. Substituting Equation (69) in Equation (65) and integrating $\boldsymbol{U}_{x}(y)$ along $y$-direction and normalizing by $H$, will result in finding a normalized volume flow rate $U_{x A}$ at $K n \gg 1$ :

$$
U_{x A}=2 \text {. }
$$

Remarkably, the normalized volume flow rate reaches a constant value for $n \rightarrow \infty$, which agrees with Knudsen's finding [29].

\section{Discussion and Conclusions}

The recently discovered and mathematically proven BPPBS [4] is used to determine the rarefied gas flow rate through the microchannel spaced between two infinite parallel plates in the current paper. Our theory does not use the generalized concept of viscosity based on the variable MFP in the KL. We formulated the Ballistic Model's physical principles for one-dimensional incompressible steady-state gas flow at the uniform temperature, a lack of external field of the force, and low flow velocity (with the Mach number less than 0.3 ). We have formulated the mass and momentum balance equations (momentum associated with the thermal component of velocity). Then we have solved them, revealing that the rate of collisions on the plates' gas-solid interface is independent of the rarefication (independent from Knudsen number). Since there is a chance for a particle diffusively scattered from one confining plate to reaching by the free path the opposite plate, we were able to derive an analytical function revealing the dependence of the effective density of particles currying $\boldsymbol{u}_{x}$-momentum from the Knudsen number. It has been found that the effective normalized density is 
constant within the gas space and depends only on the Knudsen number. Considering the effect of the confining plates on the density of $\boldsymbol{u}_{x}$-momentum carriers in the channel's gas space, we have formulated an integral form expressing implicitly the velocity profile generated in the model gas due to the pressure gradient along the channel. The implicit integral form of the velocity profile has been solved numerically by the sequential approximation. Comparing the normalized flow rate as a function of the inverse Knudsen number according to the current theory and the experimental data shows good agreement in the range of the inverse Knudsen number from 0.01 to about 40. The correlation factor is found to be about 0.995 . Also, a differential form of the $\boldsymbol{u}_{x}$-momentum balance equation governing gas flow in the channel between two parallel plates due to the pressure gradient along the channel has been derived. It was noted that the differential form of the $\boldsymbol{u}_{x}$-momentum balance equation derived from the implicit integral form of the velocity profile is different from the classical Navier-Stokes equation applicable to incompressible Newtonian flow. However, the governing equation derived in the present theory is reduced, for $K n \rightarrow 0$, to the Navier-Stokes equation for well-known general Couette flow. In turn, for $K n \rightarrow \infty$, the governing equation is reduced to a constant value, thus representing the free-molecular regime. It implies that both the integral and the differential forms, according to our theory, are applicable to describe gas flow in the microchannel in the range of Knudsen numbers from zero to infinity.

We also analyzed the validity of the currently widely disseminated claim of the MFP variation in the KL as a function of the distance from the gas-solid interface. For this purpose, we compared the rate of collisions per unit area on a gas-solid for the incompressible gas at the uniform temperature in a semi-infinite space for our and other models based on the variable MFP in the KL region. First, we found that the collision rate per unit area obtained by applying the BM and the corresponding characteristic known from the kinetic theory of gases are correlated and analytically identical. These findings support our theory based on the BM and the BPPBS. Second, we incorporated in the equation expressing the total rate of collisions per unit area on the solid surface, which we have introduced earlier in [4], the function representing the variable MFP [12]. However, in the latter case, upon executing identical algebra operations, we could not produce a meaningful value for the collisions' rate per unit area of the incompressible gas.

Based on the results above, we may conclude the following: fictitious

1) "Knudsen layer" seems to be a useful abstraction to employ specific boundary conditions providing reasonable approximate solution beyond the Knudsen layer (by using Navier-Stokes equations or a lattice Boltzmann (LB) method to model a rarefied gas flow). However, gas particles' microscopic and physical behavior in the KL is not well understood, leading to a pure prediction of gas flow characteristics.

2) The assumption that the MFP varies as a function of the surface confinement (Knudsen number), widely disseminated in the literature, is erroneous. 
Such an assumption disagrees with the kinetic theory of gases. As we found, the interaction by collisions of the gas with variation in MFP does not produce a meaningful value for the rate of collisions per unit area. As a result, such an assumption leads to wrong physical interpretations of nanoscale gas flows.

3) We offer a new way of computing a steady-state rarefied gas flow in a microchannel based on recently discovered and mathematically proven by us the Ballistic Principle of the Property Balance in Space. We specifically investigated the mechanism of influence of the Knudsen number on the rarefied gas flow. Our theory does not use the generalized concept of viscosity based on the variable MFP in the KL. Comparing the normalized flow rate as a function of the inverse Knudsen number according to the current theory and the experimental data shows good agreement in the range of the inverse Knudsen number from 0.01 to about 40 . The correlation factor is found to be about 0.995 .

4) Based on the BPPBS, our theory is advantageous over any available methods or theories for modeling rarefied gas flow. It allows the formulation of the integro-differential or integral form of the property balance (mass, momentum, energy, or any other property) and obtaining an exact solution of the rarefied gas problem. Our approach based on the BPPBS is computationally efficient. It offers substantial and practical advantages in modeling and simulation of rarefied gases.

\section{Conflicts of Interest}

The author declares no conflicts of interest regarding the publication of this paper.

\section{References}

[1] Wang, W., Jia, J. and Li, J. (2013) Slide Film Damping in Microelectromechanical System Devices. Proceedings of the Institution of Mechanical Engineers, Part N: Journal of Nanoengineering and Nanosystems, 227, 162-170. https://doi.org/10.1177/1740349913486097

[2] Tang, G., He, Y. and Tao, W. (2007) Comparison of Gas Slip Models with Solutions of Linearized Boltzmann Equation and Direct Simulation of Monte Carlo Method. International Journal of Modern Physics C, 18, 203-216. https://doi.org/10.1142/S0129183107010383

[3] Guo, Z.L., Shi, B.C. and Zheng, C.G. (2007) An Extended Navier-Stokes Formulation for Gas Flows in the Knudsen Layer near a Wall. EPL, 80, 24001. https://doi.org/10.1209/0295-5075/80/24001

[4] Kislov, N. (2020) Ballistic Principle of the Property Balance in Space and Its Application to Modeling of Fluid Dynamics Problems. Journal of Applied Mathematics and Physics, 8, 1081-1122. https://doi.org/10.4236/jamp.2020.86084

[5] Zhang, Y.H., Gu, X.J., Barber, R.W. and Emerson, D.R. (2006) Capturing Knudsen Layer Phenomena Using a Lattice Boltzmann Model. Physical Review E, Statistical, Nonlinear, and Soft Matter Physics, 74, Article ID: 046704. https://doi.org/10.1103/PhysRevE.74.046704

[6] Dongari, N., Zhang, Y. and Reese, J. (2010) The Importance of Mean Free Path in Determining Gas Micro Flow Behaviour. In: 8th International Conference on Na- 
nochannels, Microchannels and Minichannels, Montréal, 1-5 August 2010, 481-490. http://strathprints.strath.ac.uk https://doi.org/10.1115/FEDSM-ICNMM2010-30743

[7] Arlemark, E.J., Dadzie, S.K. and Reese, J.M. (2010) An Extension to the Navier-Stokes Equations to Incorporate Gas Molecular Collisions with Boundaries. Journal of Heat Transfer, 132, Article ID: 041006. https://doi.org/10.1115/1.4000877

[8] Perumanath, S., Prabha, S.K. and Sathian, S.P. (2015) The Effect of Characteristic Length on Mean Free Path for Confined Gases. Physica A, 437, 68-74.

https://doi.org/10.1016/j.physa.2015.05.022

[9] Xie, J., Borg, M.K., Gibelli, L., Henrich, O., Lockerby, D.A. and Reese, J.M. (2019) Effective Mean Free Path and Viscosity of Confined Gases. Physics of Fluids, 31, Article ID: 072002. https://doi.org/10.1063/1.5108627

[10] To, Q.D., Léonard, C. and Lauriat, G. (2015) Free-Path Distribution and Knudsen Layer Modeling for Gaseous Flows in the Transition Regime. Physical Review E, 91, Article ID: 023015. https://doi.org/10.1103/PhysRevE.91.023015

[11] Wikipedia (2020) Molecular Dynamics. https://en.wikipedia.org/wiki/Molecular_dynamics

[12] Abramov, V. (2018) Gas near a Wall: Shortened Mean Free Path, Reduced Viscosity, and the Manifestation of the Knudsen Layer in the Navier-Stokes Solution of a Shear Flow. Journal of Nonlinear Science, 28, 833-845. https://doi.org/10.1007/s00332-017-9429-7

[13] Stops, D.W. (1970) The Mean Free Path of Gas Molecules in the Transition Regime. Journal of Physics D: Applied Physics, 3, 685-696. https://doi.org/10.1088/0022-3727/3/5/307

[14] Hadjiconstantinou, N. (2006) The Limits of Navier-Stokes Theory and Kinetic Extensions for Describing Small-Scale Gaseous Hydrodynamic. Physics of Fluids, 18, Article ID: 111301. https://doi.org/10.1063/1.2393436

[15] Barisik, M. and Beskok, A. (2015) Molecular Free Paths in Nanoscale Gas Flows. Microfluid Nanofluid, 18, 1365-1371. https://doi.org/10.1007/s10404-014-1535-3

[16] Dongari, N., Sharma, A. and Durst, F. (2009) Pressure-Driven Diffusive Gas Flows in Micro-Channels: From the Knudsen to the Continuum Regimes. Microfluid Nanofluid, 6, 679-692. https://link.springer.com/10.1007/s10404-008-0344-y https://doi.org/10.1007/s10404-008-0344-y

[17] Durst, F., Gomes, J. and Sambasivam, R. (2006) Thermofluiddynamics: Do We Solve the Right Kind of Equations? Proceeding of the International Symposium on Turbulence, Heat and Mass Transfer, Dubrovnik, 3-18 September 2006, 25-29. https://doi.org/10.1615/ICHMT.2006.TurbulHeatMassTransf.10

[18] Kislov, N. (2020) Novel Analytical Molecular Dynamics Technique for Solving Fluid Dynamics Problems, Chapter 1. In: Theory and Practice of Mathematics and Computer Science, Vol. 5, Book Publisher International, India, United Kingdom, 1-41. https://doi.org/10.9734/bpi/tpmcs/v5

[19] Ohwada, T., Sone, Y. and Aoki, K. (1989) Numerical Analysis of the Poiseuille and Thermal Transpiration Flows between Parallel Plates on the Basis of the Boltzmann Equation for Hard-Sphere Molecules. Physics of Fluids A: Fluid Dynamics, 1, 2042-2049. https://doi.org/10.1063/1.857478

[20] Shen, S., Chen, G., Crone, R.M. and Anaya-Dufresne, M. (2007) A Kinetic-Theory Based First Order Slip Boundary Condition for Gas Flow. Physics of Fluids, 19, Article ID: 086101. https://doi.org/10.1063/1.2754373

[21] Lockerby, D., Reese, J., Emerson, D. and Barber, R. (2004) Velocity Boundary Con- 
dition at Solid Walls in Rarefied Gas Calculations. Physical Review E, 70, Article ID: 017303. https://doi.org/10.1103/PhysRevE.70.017303

[22] Li, Q., He, Y., Tang, G. and Tao, W. (2011) Lattice Boltzmann Modeling of Microchannel Flows in the Transition Flow Regime. Microfluidics and Nanofluidics, 10, 607-618. https://doi.org/10.1007/s10404-010-0693-1

[23] Cercignany, C. and Lorenzani, S. (2010) Variational Derivation of Second-Order Slip Coefficients on the Basis of the Boltzmann Equation for Hard-Sphere Molecules. Physics of Fluids, 22, Article ID: 062004. https://doi.org/10.1063/1.3435343

[24] Zhang, W., Meng, G. and Wei, X. (2012) A Review on Slip Models for Gas Microflows. Microfluidics and Nanofluidics, 13, 845-882.

https://doi.org/10.1007/s10404-012-1012-9

[25] Kislov, N. (2019) Analytical Tools and Methods for Modeling Transport Processes in Fluids. US Patent No. 10467362.

[26] Borman, V.D., Krylov, S.Yu. and Prosyanov, A.V. (1988) Theory of Nonequilibrium Phenomena at a Gas-Solid Interface. Journal of Experimental and Theoretical Physics, 67, 2110-2121. http://www.jetp.ac.ru/cgi-bin/dn/e_067_10_2110.pdf

[27] Kanki, T. and Iuchi, S. (1973) Poiseuille Flow and Thermal Creep of a Rarefied Gas between Parallel Plates. Physics of Fluids, 16, 594. https://doi.org/10.1063/1.1694393

[28] Loyalka, S.K. (1974) Comments on Poiseuille Flow and Thermal Creep of a Rarefied Gas between Parallel Plates. Physics of Fluids, 17, 1053-1055.

https://doi.org/10.1063/1.1694820

[29] Knudsen, M. (1909) Die Gesetze der Molekularstromung und der inneren Reibungsstromung der Gase durch Rohren (The Laws of Molecular and Viscous Flow of Gases through Tubes). Annals of Physics, 333, 75-130. https://doi.org/10.1002/andp.19093330106

[30] Polyanin, A.D. and Manzhirov, A. (2008) Handbook of Integral Equations. 2nd Edition, CRC, Chapman and Hall, London, 564-565.

https://doi.org/10.1201/9781420010558 